\title{
VANISHING CONDITIONS FOR THE SIMPLICIAL VOLUME OF COMPACT COMPLEX VARIETIES
}

\author{
MARINA VILLE
}

(Communicated by Peter Li)

\begin{abstract}
Gromov has defined a notion of simplicial volume: it is a topological invariant for compact manifolds which is closely related to the fundamental group. We investigate here the relevance of this notion in the realm of complex varieties.
\end{abstract}

\section{INTRODUCTION}

1. Statement of the results. The simplicial volume of a manifold $X$ measures the smallest number of simplices necessary to express the fundamental homology class of $X$; it is denoted by $\|X\|$. It has been defined and studied by Gromov in [Gro] who shows, among other things, that $\|X\|$ vanishes if $\Pi_{1}(X)$ is amenable. On the other hand, if $X$ admits a metric of negative sectional curvature, then $\|X\| \neq 0$. In the present paper we ask the following question:

If $X$ is a compact complex manifold with nonzero simplicial volume, what consequences can we derive in terms of its complex structure?

The general theory of [Gro] tells us that if $f: X \rightarrow Y$ is a degree 1 map which induces an isomorphism between the fundamental groups, then $\|X\|=\|Y\|$. So the simplicial volume is a bimeromorphic invariant between smooth complex manifolds.

Remark. The simplicial volume of singular complex varieties can also be defined (see [Gro]), but it is not a bimeromorphic invariant.

Before stating the results we need to recall the following.

Notation. If $A$ is a subset of a topological space $B$, we will denote by $i_{*}$ the natural map $\Pi_{1}(A) \rightarrow \Pi_{1}(B)$ induced by the inclusion $i: A \subset B$.

Definition 1 ([Gro]). A subset $Y \subset X$ of a topological space $X$ is called amenable in $X$ if for every path-connected component $Y^{\prime}$ of $Y$, the image of the inclusion homomorphism $i_{*}: \Pi_{1}\left(Y^{\prime}\right) \rightarrow \Pi_{1}(X)$ is an amenable subgroup of $\Pi_{1}(X)$.

We will prove the following:

Received by the editors July 31, 1992 and, in revised form, September 30, 1993.

1991 Mathematics Subject Classification. Primary 14C20, 14D05, 14F35, 14F45, 20F32, 32S50, $55 \mathrm{~N} 35$.

Key words and phrases. Simplicial volume, complex variety, fundamental group, bounded cohomology. 
Theorem 1. Let $X$ be a compact complex manifold, let $S$ be a (possibly singular) projective variety, and let $\left(D_{s}\right)_{s \in S}$ be an analytic family of divisors such that:

(i) $\forall x \in X, \exists s \in S$ such that $x \in D_{s}$ and

(ii) $\exists s_{0} \in S$ such that $D_{s_{0}}$ is amenable in $X$. Then $\|X\|=0$.

Remark. The $D_{s}$ 's are not a priori supposed to be smooth, so "analytic family" should be understood, for instance, in the space of cycles sense of the words (see [Ba] for instance).

In the same range of ideas, it is not difficult to derive from the classification theory of surfaces and threefolds the

Proposition 1. Let $X$ be a compact Kähler manifold of complex dimension n, $n \leq 3$, such that $\|X\| \neq 0$. Then:

(i) If $n=2$, then $X$ is projective of general type.

(ii) If $n=3$, one of the following two cases occur:

(a) $X$ is projective of general type, or

(b) $a(X)=0$ and $b_{1}(X)=0$, where $a(X)$ denotes the algebraic dimension of $X$ and $b_{1}(X)$ its first Betti number.

Finally we prove

Proposition 2. Let $X$ be a compact complex manifold with nonzero simplicial volume, and let $L$ be a linear system on $X$ of strictly positive dimension. Then $L$ has at least one singular element.

2. A motivation for Theorem 1. Theorem 1 can be seen as a "Lefschetz type" result. Lefschetz's theorem tells us, among other things, that if a projective manifold $X$ has a simply-connected smooth hyperplane section, then $X$ is itself simplyconnected. Now relax the assumption on $D$ and only assume that $D$ belongs to an analytic family of dimension $\geq 1$. Then Theorem 1 tells us then that, although $X$ may itself not be simply connected any more, at least there is one $\Pi_{1}$-related invariant of $X$, namely the simplicial volume of $X$, which vanishes.

\section{Preliminaries to the proof of Theorem 1}

We need to recall some facts which we will use in our proof of Theorem 1.

1. Some dimension theory. We will need the following fact of dimension theory (it is actually the definition of the dimension of a topological space).

Fact $1([\mathrm{Hu}-\mathrm{Wa}])$. Let $X$ be a CW-complex of dimension $n$. Then there exists a covering of $X$ by a family of open contractible sets $\left(U_{i}\right)$, such that the intersection of any family of $n+1$ different $U_{i}$ 's is empty.

2. Simplicial volume and bounded cohomology. The reference for what follows is [Gro].

Let $X$ be a topological space. A singular $p$-cocycle $c$ on $X$ is said to be bounded if there exists a positive number $A$ such that for every $p$-simplex $\sigma$ on $X$ we have

$$
|c(\sigma)| \leq A \text {. }
$$

The bounded cocycles form a complex $\widehat{C}^{*}(X, \mathbb{R})$, the differential being the usual differential in singular cohomology; the homology $\widehat{H}^{*}(X, \mathbb{R})$ of this complex is called the bounded cohomology of $X$. 
If $H^{*}(X, \mathbb{R})$ denotes the usual singular cohomology of $X$, there is an obvious natural map

$$
\widehat{H}^{*}(X, \mathbb{R}) \rightarrow H^{*}(X, \mathbb{R}) .
$$

A usual singular cohomology class is said to be bounded if it can be represented by a bounded cocycle; in other words if it belongs to the image of the mapping

$$
\widehat{H}^{*}(X, \mathbb{R}) \rightarrow H^{*}(X, \mathbb{R}) .
$$

Gromov shows (using the Hahn-Banach theorem) that an orientable compact manifold has nonzero simplicial volume if and only if its fundamental orientation class is bounded.

3. Bounded cohomology and amenability. Gromov shows that a topological space with an amenable fundamental group has trivial bounded cohomology. He then goes on to prove the following finer fact.

Theorem 2. Let $X$ be a topological space, let $p$ be a positive integer, and suppose there is a covering of $X$ by a family of open sets $\left(U_{i}\right)$ such that

(i) each $U_{i}$ is amenable in $X$ and

(ii) the intersection of any family of $p U_{i}$ 's is empty.

Then for every integer $k \geq p$, the map

$$
\widehat{H}^{k}(X, \mathbb{R}) \rightarrow H^{k}(X, \mathbb{R})
$$

has zero image.

Gromov gets from Theorem 2 the following corollary.

Theorem 3. Let $X$ be a topological space, let $Y$ be a manifold of dimension $p \in \mathbb{N}$, and suppose there exists a continuous map

$$
f: X \rightarrow Y
$$

such that the preimage of every element of $Y$ is amenable in $X$. Then for every $k \geq p+1$, the map

$$
\widehat{H}^{k}(X, \mathbb{R}) \rightarrow H^{k}(X, \mathbb{R})
$$

has zero image.

Theorem 3 is derived from Theorem 2 via the above-mentioned Fact 1 .

Indeed, let $\left(U_{i}\right)$ be a covering of $Y$ such that the intersection of any family of $p$ different $U_{i}$ 's is empty. Then $\left(p^{-1}\left(U_{i}\right)\right)$ is a covering of $X$ satisfying the assumption of Theorem 2; and Theorem 3 follows.

We are now ready for the

4. Sketch of the proof of Theorem 1. It is analogous to the proof of Theorem 3 which we have just recalled. Namely, we will find a map from $X$ to something of smaller dimension (actually a projective curve $C$ ), with amenable fibres. It will not be as simple as with Theorem 3: first, to get the map from $X$ to $C$, we have to change our initial space $X$ and replace it by another space which we denote by $\Gamma$. And secondly we will not be able to get all the preimages of this map to be amenable: they will be amenable except for a finite number of them. Nevertheless we will be able to lift a covering of $C$ by contractible sets, add a few open sets around the nonamenable preimages, and get an open covering of $X$ (or rather of its 
replacement $\Gamma$ ) by amenable open sets $U_{i}$ 's such that the intersection of any family of $p$ different $U_{i}$ 's is empty.

\section{Proof of Theorem 1}

Step 1. Map $X$ onto a projective curve $C$ with many amenable fibres.

Actually, as we said, we won't map $X$ itself onto $C$ but another space $\Gamma$ closely related to $X$ and which we now describe.

Let $C$ be a (possibly singular) irreducible projective curve contained in $S$ and containing $s_{0}$. We consider the graph $\Gamma$ of $C$, namely,

$$
\Gamma=\{(x, g) \in X \times C / x \in g\} .
$$

We denote by $n$ the complex dimension of $X$.

Lemma 1. If the map

$$
\widehat{H}^{2 n}(\Gamma, \mathbb{R}) \rightarrow H^{2 n}(\Gamma, \mathbb{R})
$$

is zero, then the map

$$
\widehat{H}^{2 n}(X, \mathbb{R}) \rightarrow H^{2 n}(X, \mathbb{R})
$$

is also zero.

Proof. Let $p_{1}: \Gamma \rightarrow X$ be the first projection. We claim that the map

$$
\left(p_{1}\right)^{*}: H^{2 n}(X, \mathbb{R}) \rightarrow H^{2 n}(\Gamma, \mathbb{R})
$$

is injective. Indeed, let $\widetilde{\Gamma}$ be a smooth model for $\Gamma$; then we have a chain of holomorphic maps

$$
\widetilde{\Gamma} \rightarrow \Gamma \rightarrow X
$$

which are almost everywhere one-to-one.

$\widetilde{\Gamma}$ and $X$ are smooth compact $2 n$-dimensional manifolds and the composed map $\widetilde{\Gamma} \rightarrow X$ is of degree one, so the composed map

$$
H^{2 n}(X, \mathbb{R}) \stackrel{\left(p_{1}\right)^{*}}{\longrightarrow} H^{2 n}(\Gamma, \mathbb{R}) \rightarrow H^{2 n}(\widetilde{\Gamma}, \mathbb{R})
$$

is injective, so $\left(p_{1}\right)^{*}$ is also injective.

The lemma now follows from the commutative diagram

$$
\begin{gathered}
\hat{H}^{2 n}(X, \mathbb{R}) \rightarrow H^{2 n}(X, \mathbb{R}) \\
\downarrow \quad \downarrow \\
\hat{H}^{2 n}(\Gamma, \mathbb{R}) \rightarrow H^{2 n}(\Gamma, \mathbb{R})
\end{gathered}
$$

We consider now the second projection $p: \Gamma \rightarrow C$. It is a holomorphic map; we are going to show (Lemmas 2 and 3 ) that only its singular values, say $s_{0}, s_{1}, \ldots, s_{n}$, can have a preimage which is not amenable in $\Gamma$.

Lemma 2. There exists a finite subset $\Sigma=\left\{s_{0}, s_{1}, \ldots, s_{n}\right\} \subset C$ such that if $U=$ $C-\Sigma$, the restriction

$$
\left.p\right|_{p^{-1}(U)}: p^{-1}(U) \rightarrow U
$$

is a locally trivial smooth fibration. 
If $\Gamma$ is smooth, this is elementary (we take the $s_{i}$ 's to be the singular values of $p$ ). In the general case, we consider a finite Whitney stratification ([Go- $\mathrm{McPh}], \mathrm{pp}$. 37 and 43) of $\Gamma$ and we take the $s_{i}$ 's to be

(i) the images of the strata which are reduced to a point,

(ii) the singular values of the restriction of $p$ to the various strata.

Then Lemma 2 follows from Thom's first isotopy lemma (see [Go-McPh], I.1.15, and the references there).

Lemma 3. Let $u \in U$. Then $p^{-1}(u)$ is amenable in $\Gamma$.

Proof. We know $([\mathrm{Lj}])$ that $p^{-1}\left(s_{0}\right)$ admits a tubular neighbourhood $\mathcal{T}_{0}$ in $\Gamma$.

So we first consider the case of an element $u_{0}$ of $U$ such that $p^{-1}\left(u_{0}\right) \subset \mathcal{T}_{0} . p^{-1}\left(u_{0}\right)$ is obviously amenable in $\Gamma$ since its inclusion in $\Gamma$ factors through its inclusion in $\mathcal{T}_{0}$; and $\mathcal{T}_{0}$ is amenable in $\Gamma$.

If $u$ is a general element of $U$, we can parallel transport (w.r.t. the fibration $\left.p^{-1}(U) \rightarrow U\right)$ the fibre $p^{-1}(u)$ onto $p^{-1}\left(u_{0}\right)$; and so $p^{-1}(u)$ is also amenable in $\Gamma$.

Step 2. Construct the desired open cover $\left(U_{i}\right)$ such that every family of $2 n U_{i}$ 's has empty intersection.

Notation. We consider a distance $d$ on $C$; if $x$ is a point in $C$ and $r$ is a positive real number, we denote by $B(x, r)$ the open ball in $C$ centered in $x$ of radius $r$.

We are going to define three families of open sets in $\Gamma$ which, put together, will cover $\Gamma$.

First family. It covers a big open set of regular points (for $p$ ) in $\Gamma$. We choose a positive, small enough real number $\varepsilon$; removing a ball of radius $\varepsilon$ around each singular value $s_{k}$, we consider the open set in $C, V=C \backslash \bigcup B\left(s_{k}, \varepsilon\right) . \quad V$ is of dimension 2, so, using Fact 1, we cover $V$ with a family of contractible open sets $\left(V_{i}\right)$ such that every subfamily of 3 different $V_{i}$ 's has empty intersection. Pulling back the $V_{i}$ 's we get a cover of $p^{-1}(V)$ by the $p^{-1}\left(V_{i}\right)$ 's with the following properties:

(i) the $p^{-1}\left(V_{i}\right)$ 's are amenable (Lemma 2 ) and

(ii) the intersection of any subfamily of 3 different $p^{-1}\left(V_{i}\right)$ 's is empty.

Second family. It covers a neighbourhood of the singular fibres.

For each singular value $s_{k}$, we consider a small enough tubular neighbourhood $\mathcal{T}_{k}$ of the singular fibre $p^{-1}\left(s_{k}\right)$ (small enough means here that $\left.p\left(\mathcal{T}_{k}\right) \subset B\left(s_{k}, \varepsilon\right)\right)$.

$p^{-1}\left(s_{k}\right)$ is an $(n-1)$-(complex) dimensional analytic space, so it is a (real dimensional) $2 n-2 \mathrm{CW}$-complex; so, following Fact 1 , we can cover $p^{-1}\left(s_{k}\right)$ and hence $\mathcal{T}_{k}$ with a family of contractible open sets $\left(\mathcal{V}_{k, j}\right)_{j \in J}$ such that any subfamily of $2 n-2$ different $\mathcal{V}_{k, j}$ 's has empty intersection.

Third family. It patches the previous two families.

It is clear that, for every $k, p\left(\mathcal{T}_{k}\right)$ is a neighbourhood of $s_{k}$, hence it contains a small ball centered at $s_{k}$, say $B\left(s_{k}, \frac{1}{2} \varepsilon\right)$. So we consider the open sets

$$
W_{k}=p^{-1}\left\{x \in \Gamma / \frac{1}{2} \varepsilon<d\left(s_{k}, x\right)<2 \varepsilon\right\} .
$$

Lemma 4. For all $k, W_{k}$ is amenable in $\Gamma$.

Proof. Following Lemma $2, W_{k}$ is a fibration via $p$ over the annulus

$$
\left\{x \in \Gamma / \frac{1}{2} \varepsilon<d\left(s_{k}, x\right)<2 \varepsilon\right\} .
$$




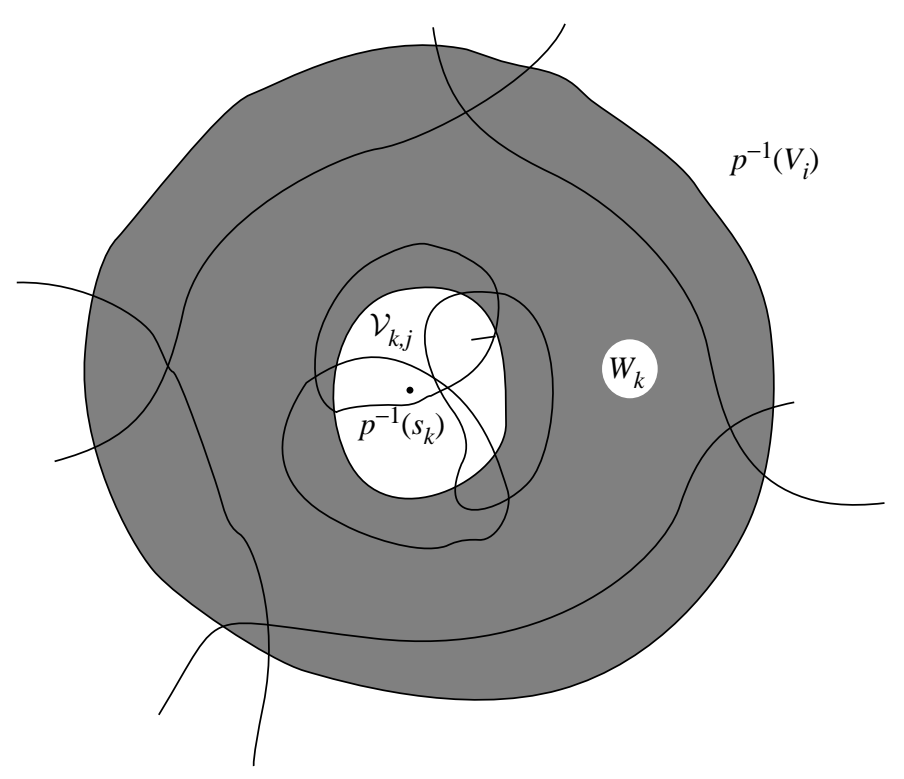

Figure 1

The fibre is amenable in $\Gamma$; the fundamental group of the base (the annulus) is $\mathbb{Z}$, hence it is amenable. So Lemma 3 follows from the exact homotopy sequence of a fibration (we recall that an extension of amenable groups is amenable).

We can now conclude. It is clear that the family $\mathcal{F}$ of open sets formed by the reunion of the three families described above, namely, the $p^{-1}\left(V_{i}\right)$ 's, the $\mathcal{V}_{k, j}$ 's, and the $W_{k}$ 's cover $\Gamma$ (see Figure 1 ). And we leave it to the reader to check that every intersection of $2 n$ different elements of $\mathcal{F}$ is empty.

\section{Proof of Proposition 2}

If necessary we subtract the fixed component of $L$ and/or blow up a codimension 2 base locus. So we can assume that there is a smooth fibration

$$
r: X \rightarrow \mathbb{C P}^{1},
$$

the fibres of which are the elements of $L$.

We recall the following criterion.

Theorem 4 ([Gr]). Let $M$ be a closed smooth manifold, and let

$$
f: M \rightarrow M
$$

be a map of degree $d,|d| \geq 2$. Then $\|M\|=0$.

We identify $\mathbb{C P}^{1}$ with $\mathbb{C} \cup\{\infty\}$ and define the map

$$
\begin{gathered}
\rho: \mathbb{C P}^{1} \rightarrow \mathbb{C P}^{1}, \\
\rho(z)=z^{2} \text { for } z \neq \infty, \\
\rho(\infty)=\infty,
\end{gathered}
$$

where $\rho$ is of degree 2 ; we are going to lift it to a degree 2 map

$$
\tilde{\rho}: X \rightarrow X \text {. }
$$


For each $z \in \mathbb{C P}^{1}$, we define a path $\gamma_{z}$ in $\mathbb{C P}^{1}$ :

$$
\begin{array}{ll}
\text { for } z \neq \infty & \gamma_{z}(t)=(1-t) z+t z^{2}, \\
\text { for } z=\infty & \gamma_{\infty}(t) \equiv \infty \quad \forall t \in[0,1] .
\end{array}
$$

Next, we endow $X$ with a Riemannian metric; this gives us a connection (i.e., a distribution of horizontal spaces in the tangent bundle). So, for every $x \in X$, we get a unique horizontal path $\gamma_{x}$ which projects via $r$ to $\gamma_{r(x)}$ and such that $\gamma_{x}(0)=x$.

We put

$$
\rho(x)=\gamma_{x}(1)
$$

It is clear that $\rho$ is orientation preserving; hence the degree of $\rho$ is equal to the set-theoretic number of preimages of a generic periodic $x$ in $X$. The restriction of $\rho$ to a fibre

$$
\rho: r^{-1}(z) \rightarrow r^{-1}\left(z^{2}\right)
$$

is obviously of degree 1 (it is a homotopy equivalence). So $\operatorname{deg} \rho=2$ (each point of $r^{-1}\left(z^{2}\right)$ is the target of one point in $r^{-1}(z)$ and one point in $\left.r^{-1}(-z)\right)$. Q.E.D.

\section{ACKNOWLEDGMENT}

We are indebted to $\mathrm{Y}$. Moriah for directing us to $[\mathrm{Hu}-\mathrm{Wa}]$ and to the referee for very helpful comments and suggestions.

\section{REFERENCES}

[Ba] D. Barlet, Espace analytique réduit des cycles analytiques complexes compacts d'un espace analytique complexe de dimension finie, Fonctions de Plusieurs Variables Complexes (Séminaire François Norguet 1974-75), Lecture Notes in Math., vol. 482, Springer-Verlag, Berlin and New York, 1975, pp. 1-158. MR 53:3347

[Gr] F. Greenleaf, Invariant means on topological groups, Van Nostrand \& Reinhold, Princeton, NJ, 1969. MR 40:4776

[Go-McPh] M. Goretsky and R. MacPherson, Stratified Morse Theory, Springer-Verlag, 1988.

[Gro] M. Gromov, Volume and bounded cohomology, Inst. Hautes Études Sci. Publ. Math. 56 (1982), 1-99. MR 84h:53053

[Hu-Wa W. Hurewicz and H. Wallman, Dimension theory, Princeton Univ. Press, Princeton, NJ, 1941. MR 3:312b

[Lj] S. Lojasiewicz, Triangulation of semi-analytic sets, Ann. Scuola Norm. Sup. Pisa Ser. III 18 (1964), 449-474. MR 30:3478

Centre de Mathématiques, École Polytechnique, 91128 Palaiseau Cedex, France

E-mail address: ville@orphee.polytechnique.fr 